CLNS 97/1480

CLEO 97-9

\title{
Determination of the Michel Parameters and the $\tau$ Neutrino Helicity in $\tau$ Decay
}

\author{
CLEO Collaboration
}

\begin{abstract}
Using the CLEO II detector at the $e^{+} e^{-}$storage ring CESR, we have determined the Michel parameters $\rho, \xi$, and $\delta$ in $\tau^{\mp} \rightarrow l^{\mp} \nu \bar{\nu}$ decay as well as the $\tau$ neutrino helicity parameter $h_{\nu_{\tau}}$ in $\tau^{\mp} \rightarrow \pi^{\mp} \pi^{0} \nu$ decay. From a data sample of $3.02 \times 10^{6} \tau$ pairs produced at $\sqrt{s}=10.6 \mathrm{GeV}$, using events of the topology $e^{+} e^{-} \rightarrow \tau^{+} \tau^{-} \rightarrow\left(l^{ \pm} \nu \bar{\nu}\right)\left(\pi^{\mp} \pi^{0} \nu\right)$ and $e^{+} e^{-} \rightarrow \tau^{+} \tau^{-} \rightarrow\left(\pi^{ \pm} \pi^{0} \bar{\nu}\right)\left(\pi^{\mp} \pi^{0} \nu\right)$, and the determined sign of $h_{\nu_{\tau}}$ [1,2], the combined result of the three samples is: $\rho=0.747 \pm 0.010 \pm 0.006, \xi=1.007 \pm 0.040 \pm 0.015, \xi \delta=0.745 \pm 0.026 \pm 0.009$, and $h_{\nu_{\tau}}=-0.995 \pm 0.010 \pm 0.003$. The results are in agreement with the Standard Model $V-A$ interaction.
\end{abstract}


J. P. Alexander, ${ }^{1}$ C. Bebek,${ }^{1}$ B. E. Berger,${ }^{1}$ K. Berkelman,${ }^{1}$ K. Bloom,${ }^{1}$ D. G. Cassel,${ }^{1}$ H. A. Cho, ${ }^{1}$ D. M. Coffman, ${ }^{1}$ D. S. Crowcroft,${ }^{1}$ M. Dickson, ${ }^{1}$ P. S. Drell,,${ }^{1}$ K. M. Ecklund,${ }^{1}$ R. Ehrlich, ${ }^{1}$ R. Elia, ${ }^{1}$ A. D. Foland,${ }^{1}$ P. Gaidarev, ${ }^{1}$ R. S. Galik,${ }^{1}$ B. Gittelman, ${ }^{1}$ S. W. Gray, ${ }^{1}$ D. L. Hartill, ${ }^{1}$ B. K. Heltsley, ${ }^{1}$ P. I. Hopman, ${ }^{1}$ J. Kandaswamy, ${ }^{1}$ P. C. Kim, ${ }^{1}$ D. L. Kreinick, ${ }^{1}$ T. Lee,${ }^{1}$ Y. Liu, ${ }^{1}$ G. S. Ludwig,${ }^{1}$ J. Masui, ${ }^{1}$ J. Mevissen,${ }^{1}$ N. B. Mistry,${ }^{1}$ C. R. Ng, ${ }^{1}$ E. Nordberg, ${ }^{1}$ M. Ogg, ${ }^{1}$. J. R. Patterson, ${ }^{1}$ D. Peterson, ${ }^{1}$ D. Riley,${ }^{1}$ A. Soffer ${ }^{1}$ B. Valant-Spaight, ${ }^{1}$ C. Ward,${ }^{1}$ M. Athanas,${ }^{2}$ P. Avery, ${ }^{2}$ C. D. Jones, ${ }^{2}$ M. Lohner, ${ }^{2}$ C. Prescott, ${ }^{2}$ J. Yelton, ${ }^{2}$ J. Zheng, ${ }^{2}$ G. Brandenburg, ${ }^{3}$ R. A. Briere, ${ }^{3}$ Y. S. Gao, ${ }^{3}$ D. Y.-J. Kim, ${ }^{3}$ R. Wilson, ${ }^{3}$ H. Yamamoto,${ }^{3}$ T. E. Browder, ${ }^{4}$ F. Li,${ }^{4}$ Y. Li,${ }^{4}$

J. L. Rodriguez, ${ }^{4}$ T. Bergfeld,${ }^{5}$ B. I. Eisenstein,${ }^{5}$ J. Ernst, ${ }^{5}$ G. E. Gladding, ${ }^{5}$ G. D. Gollin,${ }^{5}$

R. M. Hans, ${ }^{5}$ E. Johnson, ${ }^{5}$ I. Karliner, ${ }^{5}$ M. A. Marsh, ${ }^{5}$ M. Palmer, ${ }^{5}$ M. Selen, ${ }^{5}$

J. J. Thaler, ${ }^{5}$ K. W. Edwards, ${ }^{6}$ A. Bellerive,${ }^{7}$ R. Janicek, ${ }^{7}$ D. B. MacFarlane,${ }^{7}$

K. W. McLean, ${ }^{7}$ P. M. Patel,${ }^{7}$ A. J. Sadoff ${ }^{8}$ R. Ammar, ${ }^{9}$ P. Baringer, ${ }^{9}$ A. Bean,${ }^{9}$

D. Besson, ${ }^{9}$ D. Coppage,${ }^{9}$ C. Darling, ${ }^{9}$ R. Davis,${ }^{9}$ N. Hancock, ${ }^{9}$ S. Kotov, ${ }^{9}$ I. Kravchenko, ${ }^{9}$

N. Kwak,${ }^{9}$ S. Anderson, ${ }^{10}$ Y. Kubota,,${ }^{10}$ M. Lattery,${ }^{10}$ S. J. Lee, ${ }^{10}$ J. J. O'Neill,,${ }^{10}$

S. Patton, ${ }^{10}$ R. Poling, ${ }^{10}$ T. Riehle,${ }^{10}$ V. Savinov,${ }^{10}$ A. Smith,${ }^{10}$ M. S. Alam,${ }^{11}$ S. B. Athar, ${ }^{11}$ Z. Ling, ${ }^{11}$ A. H. Mahmood,${ }^{11}$ H. Severini,${ }^{11}$ S. Timm,${ }^{11}$ F. Wappler,${ }^{11}$ A. Anastassov, ${ }^{12}$ S. Blinov, ${ }^{12}$, J. E. Duboscq ${ }^{12}$ K. D. Fisher, ${ }^{12}$ D. Fujino, ${ }^{12}$, K. K. Gan, ${ }^{12}$ T. Hart, ${ }^{12}$ K. Honscheid,${ }^{12}$ H. Kagan,${ }^{12}$ R. Kass, ${ }^{12}$ J. Lee, ${ }^{12}$ M. B. Spencer, ${ }^{12}$ M. Sung, ${ }^{12}$ A. Undrus,${ }^{12, \dagger}$ R. Wanke, ${ }^{12}$ A. Wolf, ${ }^{12}$ M. M. Zoeller, ${ }^{12}$ B. Nemati, ${ }^{13}$ S. J. Richichi,,${ }^{13}$ W. R. Ross ${ }^{13}$ P. Skubic,${ }^{13}$ M. Wood,${ }^{13}$ M. Bishai, ${ }^{14}$ J. Fast,${ }^{14}$ E. Gerndt,${ }^{14}$ J. W. Hinson,,${ }^{14}$ N. Menon, ${ }^{14}$ D. H. Miller, ${ }^{14}$ E. I. Shibata, ${ }^{14}$ I. P. J. Shipsey, ${ }^{14}$ M. Yurko, ${ }^{14}$ L. Gibbons, ${ }^{15}$ S. Glenn ${ }^{15}$ S. D. Johnson, ${ }^{15}$ Y. Kwon,${ }^{15}$ S. Roberts, ${ }^{15}$ E. H. Thorndike, ${ }^{15}$ C. P. Jessop, ${ }^{16}$ K. Lingel,${ }^{16}$ H. Marsiske, ${ }^{16}$ M. L. Perl,${ }^{16}$ D. Ugolini,${ }^{16}$ R. Wang,${ }^{16}$ X. Zhou, ${ }^{16}$ T. E. Coan, ${ }^{17}$ V. Fadeyev ${ }^{17}$ I. Korolkov, ${ }^{17}$ Y. Maravin, ${ }^{17}$ I. Narsky, ${ }^{17}$ V. Shelkov ${ }^{17}$ J. Staeck, ${ }^{17}$ R. Stroynowski ${ }^{17}$ I. Volobouev ${ }^{17}$ J. Ye ${ }^{17}$ M. Artuso, ${ }^{18}$ A. Efimov, ${ }^{18}$ F. Frasconi, ${ }^{18}$ M. Gao, ${ }^{18}$ M. Goldberg, ${ }^{18}$ D. He, ${ }^{18}$ S. Kopp, ${ }^{18}$ G. C. Moneti, ${ }^{18}$ R. Mountain, ${ }^{18}$ S. Schuh,${ }^{18}$ T. Skwarnicki ${ }^{18}$ S. Stone, ${ }^{18}$ G. Viehhauser ${ }^{18}$ X. Xing, ${ }^{18}$ J. Bartelt,${ }^{19}$ S. E. Csorna,${ }^{19}$ V. Jain, ${ }^{19}$ S. Marka, ${ }^{19}$ R. Godang, ${ }^{20}$ K. Kinoshita,${ }^{20}$ I. C. Lai,${ }^{20}$ P. Pomianowski, ${ }^{20}$ S. Schrenk ${ }^{20}$ G. Bonvicini, ${ }^{21}$ D. Cinabro, ${ }^{21}$ R. Greene, ${ }^{21}$ L. P. Perera,${ }^{21}$ G. J. Zhou ${ }^{21}$ B. Barish, ${ }^{22}$ M. Chadha,${ }^{22}$ S. Chan,${ }^{22}$ G. Eigen,${ }^{22}$ J. S. Miller, ${ }^{22}$ C. O'Grady,${ }^{22}$ M. Schmidtler, ${ }^{22}$ J. Urheim, ${ }^{22}$ A. J. Weinstein, ${ }^{22}$ F. Würthwein, ${ }^{22}$ D. M. Asner, ${ }^{23}$ D. W. Bliss,${ }^{23}$ W. S. Brower ${ }^{23}$ G. Masek,${ }^{23}$ H. P. Paar, ${ }^{23}$ S. Prell,${ }^{23}$ V. Sharma,${ }^{23}$ J. Gronberg, ${ }^{24}$ T. S. Hill,${ }^{24}$ R. Kutschke,${ }^{24}$ D. J. Lange,${ }^{24}$ S. Menary,${ }^{24}$ R. J. Morrison,${ }^{24}$ H. N. Nelson, ${ }^{24}$ T. K. Nelson, ${ }^{24}$ C. Qiao,${ }^{24}$ J. D. Richman, ${ }^{24}$ D. Roberts,${ }^{24}$ A. Ryd,${ }^{24}$ M. S. Witherell, ${ }^{24}$ R. Balest, ${ }^{25}$ B. H. Behrens, ${ }^{25}$ K. Cho, ${ }^{25}$ W. T. Ford ${ }^{25}$ H. Park,${ }^{25}$ P. Rankin, ${ }^{25}$ J. Roy ${ }^{25}$ and J. G. Smith ${ }^{25}$

${ }^{*}$ Permanent address: University of Texas, Austin TX 78712

†Permanent address: BINP, RU-630090 Novosibirsk, Russia.

${ }^{\ddagger}$ Permanent address: Lawrence Livermore National Laboratory, Livermore, CA 94551. 


\author{
${ }^{1}$ Cornell University, Ithaca, New York 14853 \\ ${ }^{2}$ University of Florida, Gainesville, Florida 32611 \\ ${ }^{3}$ Harvard University, Cambridge, Massachusetts 02138 \\ ${ }^{4}$ University of Hawaii at Manoa, Honolulu, Hawaii 96822 \\ ${ }^{5}$ University of Illinois, Champaign-Urbana, Illinois 61801 \\ ${ }^{6}$ Carleton University, Ottawa, Ontario, Canada K1S 5B6 \\ and the Institute of Particle Physics, Canada \\ ${ }^{7}$ McGill University, Montréal, Québec, Canada H3A 2T8 \\ and the Institute of Particle Physics, Canada \\ ${ }^{8}$ Ithaca College, Ithaca, New York 14850 \\ ${ }^{9}$ University of Kansas, Lawrence, Kansas 66045 \\ ${ }^{10}$ University of Minnesota, Minneapolis, Minnesota 55455 \\ ${ }^{11}$ State University of New York at Albany, Albany, New York 12222 \\ ${ }^{12}$ Ohio State University, Columbus, Ohio 43210 \\ ${ }^{13}$ University of Oklahoma, Norman, Oklahoma 73019 \\ ${ }^{14}$ Purdue University, West Lafayette, Indiana 47907 \\ ${ }^{15}$ University of Rochester, Rochester, New York 14627 \\ ${ }^{16}$ Stanford Linear Accelerator Center, Stanford University, Stanford, California 94309 \\ ${ }^{17}$ Southern Methodist University, Dallas, Texas 75275 \\ ${ }^{18}$ Syracuse University, Syracuse, New York 13244 \\ ${ }^{19}$ Vanderbilt University, Nashville, Tennessee 37235 \\ ${ }^{20}$ Virginia Polytechnic Institute and State University, Blacksburg, Virginia 24061 \\ ${ }^{21}$ Wayne State University, Detroit, Michigan 48202 \\ ${ }^{22}$ California Institute of Technology, Pasadena, California 91125 \\ ${ }^{23}$ University of California, San Diego, La Jolla, California 92093 \\ ${ }^{24}$ University of California, Santa Barbara, California 93106 \\ ${ }^{25}$ University of Colorado, Boulder, Colorado 80309-0390
}




\section{INTRODUCTION}

The most general, local, derivative-free, and lepton-number-conserving four fermion point interaction [3 5] for leptonic $\tau$ decays yields in the helicity projection form [6] the following matrix element

$$
\mathcal{M}=4 \frac{G_{l}}{\sqrt{2}} \sum_{\substack{\gamma=S, V, T \\ \epsilon, \mu=R, L}} g_{\epsilon \mu}^{\gamma}\left[\bar{u}_{\epsilon}\left(l^{-}\right) \Gamma_{\gamma} v_{j}\left(\bar{\nu}_{l}\right)\right]\left[\bar{u}_{i}\left(\nu_{\tau}\right) \Gamma^{\gamma} u_{\mu}\left(\tau^{-}\right)\right]
$$

where $G_{l}$ parametrizes the total strength of the interaction. The matrices $\Gamma_{\gamma}$ define the properties of the two currents under a Lorentz transformation with $\gamma=S, V, T$ for scalar, vector, and tensor interactions. The indices $\epsilon$ and $\mu$ label the right- or lefthandedness (R,L) of the charged leptons. For a given $\epsilon, \mu$, and $\gamma$, the handedness of the neutrinos labeled by $j$ and $i$ are fixed. Only ten of the twelve complex coupling constants $g_{\epsilon \mu}^{\gamma}$ are linearly independent. In the Standard Model $V-A$ interaction, the only non-zero coupling constant is $g_{L L}^{V}=1$.

The interaction described by Eqn. 1 is fully determined by 19 real parameters. Without measuring the neutrinos and the spin of the outgoing charged lepton, only the four Michel parameters [3] 5] $\rho, \eta, \xi$, and $\delta$ are experimentally accessible. They are bilinear combinations of the coupling constants $g_{\epsilon \mu}^{\gamma}$ and appear in the predicted energy spectrum of the charged lepton $l^{\mp}$ emitted in the decay $\tau^{\mp} \rightarrow l^{\mp} \nu \bar{\nu}$. In the $\tau$ rest frame, neglecting radiative corrections and terms proportional to $m_{l}^{2} / m_{\tau}^{2}$, this spectrum is given by

$$
\begin{aligned}
\frac{d \Gamma\left(\tau^{\mp} \rightarrow l^{\mp} \nu \bar{\nu}\right)}{d \Omega d x}= & \frac{G_{F}^{2} m_{\tau}^{5}}{192 \pi^{4}} x^{2}\left[3(1-x)+\frac{2}{3} \rho(4 x-3)+6 \eta \frac{m_{l}}{m_{\tau}} \frac{1-x}{x}\right. \\
& \left.\mp \xi P_{\tau} \cos \theta\left((1-x)+\frac{2}{3} \delta(4 x-3)\right)\right]
\end{aligned}
$$

where $x=2 E_{l} / m_{\tau}$ is the scaled charged lepton energy, $P_{\tau}$ the $\tau$ polarization, and $\theta$ the angle between $\tau$ spin and lepton momentum. In the Standard Model the $V-A$ charged weak current is characterized by $\rho=3 / 4, \eta=0, \xi=1$ and $\delta=3 / 4$.

A measurement of the Michel parameters allows one to limit the coupling constants $g_{\epsilon \mu}^{\gamma}$. For example $\xi$ and $\delta$ determine the probability, $P_{R}^{\tau}$, for a righthanded $\tau$ lepton to participate in leptonic $\tau$ decays:

$$
P_{R}^{\tau}=\frac{1}{4}\left|g_{R R}^{S}\right|^{2}+\frac{1}{4}\left|g_{L R}^{S}\right|^{2}+\left|g_{R R}^{V}\right|^{2}+\left|g_{L R}^{V}\right|^{2}+3\left|g_{L R}^{T}\right|^{2}=\frac{1}{2}\left[1+\frac{1}{9}(3 \xi-16 \xi \delta)\right]
$$

Despite the progress made in recent years [1],2,77 [16], the determination of the space-time structure in leptonic and semihadronic $\tau$ decays is still an order of magnitude less precise than in $\mu$ decay, indicating a need for high precision measurements of the Michel parameters in leptonic $\tau$ decays as well as of the $\tau$ neutrino helicity in semihadronic $\tau$ decays. In this publication, we present measurements of $\rho, \xi, \delta$, and the $\tau$ neutrino helicity $h_{\nu_{\tau}}$ from an analysis of $e^{+} e^{-} \rightarrow \tau^{+} \tau^{-} \rightarrow\left(l^{ \pm} \nu \bar{\nu}\right)\left(\pi^{\mp} \pi^{0} \nu\right)$ and $\left(\pi^{ \pm} \pi^{0} \bar{\nu}\right)\left(\pi^{\mp} \pi^{0} \nu\right)$ events. The parameter $h_{\nu_{\tau}}$ is given by $h_{\nu_{\tau}}=2 g_{V} g_{A} /\left(g_{V}^{2}+g_{A}^{2}\right)$, where $g_{V}$ and $g_{A}$ are the vector and axialvector couplings. In the Standard Model, with purely lefthanded neutrinos, one expects $h_{\nu_{\tau}}=-1$. 
The $\left(l^{ \pm} \nu \bar{\nu}\right)\left(\pi^{\mp} \pi^{0} \nu\right)$ sample used here is correlated with that of ref. [15]. There, the Michel parameters $\rho$ and $\eta$ have been determined with emphasis on a precise measurement of $\eta$, in which the sensitivity comes mainly from the low momentum part of the muon spectrum. Here, the emphasis lies on the determination of the spin dependent Michel parameters $\xi$ and $\delta$.

\section{METHOD OF THE MEASUREMENT}

Eqn. 2 shows that the measurement of $\xi$ and $\delta$ requires the knowledge of the $\tau$ spin orientation. In $e^{+} e^{-}$annihilation at $\sqrt{s} \approx 10 \mathrm{GeV}$ the average $\tau$ polarization is zero and no information on $\xi$ and $\delta$ can be obtained from single $\tau$ decays. However, spin-spin correlations exist between the two $\tau$ leptons in $e^{+} e^{-} \rightarrow \tau^{+} \tau^{-}$, leading to correlations between kinematical properties of the decay products. These correlations have been used before [2,8.10] for the determination of $\xi, \delta$, and $h_{\nu_{\tau}}$, where leptonic as well as semihadronic decays served as spin analyzers. Here we use the semihadronic $\tau$ decay $\tau^{\mp} \rightarrow \pi^{\mp} \pi^{0} \nu$ as spin analyzer. Its advantages are a large branching ratio, a very well understood hadronic current, and an experimentally clean signal.

In the Born approximation, the matrix element for the differential cross section of $e^{+} e^{-} \rightarrow \tau^{+} \tau^{-} \rightarrow\left(l^{ \pm} \nu \bar{\nu}\right)\left(\pi^{\mp} \pi^{0} \nu\right)$ has, after integration over the unobserved neutrino degrees of freedom and summation over unobserved spins, the following structure (see for example ref. [17]):

$$
|\mathcal{M}|^{2}=H P\left[L_{1}+\rho L_{2}+\eta L_{3}\right]+h_{\nu_{\tau}} H_{\alpha}^{\prime} C^{\alpha \beta}\left[\xi L_{1 \beta}^{\prime}+\xi \delta L_{2 \beta}^{\prime}\right] .
$$

The first term is the spin averaged part of the differential cross section. The second term contains the spin correlation. For the semihadronic decay $\tau^{\mp} \rightarrow \pi^{\mp} \pi^{0} \nu$ the matrix element is formulated for an arbitrary mixing of $V$ and $A$ couplings, parametrized by the neutrino helicity $h_{\nu_{\tau}}$. The spin averaged part of this matrix element is indicated by $H$ and the spin dependent part by $H^{\prime}$. The symbols $L_{i}$ and $L_{i}^{\prime}$ are the Lorentz invariant formulations of the corresponding terms in Eqn. 2. The spin averaged $\tau$ pair production is denoted by $P$ and the production spin correlation matrix by $C^{\alpha \beta}$.

The spin analyzer $\tau^{\mp} \rightarrow \pi^{\mp} \pi^{0} \nu$ can resolve the ratio of longitudinal to transverse polarization of the intermediate $\rho$ meson, but, because of the absence of interference terms, cannot separate its transverse polarization into the left and righthanded part. Thus, our $\left(l^{ \pm} \nu \bar{\nu}\right)\left(\pi^{\mp} \pi^{0} \nu\right)$ events are sensitive to $\rho, \eta, h_{\nu_{\tau}} \xi$, and $h_{\nu_{\tau}} \xi \delta$ (see Eqn. 四), whereas our $\left(\pi^{ \pm} \pi^{0} \bar{\nu}\right)\left(\pi^{\mp} \pi^{0} \nu\right)$ events allow a determination of the product $h_{\nu_{\tau}}^{2}$. The signs of $\xi$ and $h_{\nu_{\tau}}$ are well known from other experiments [1,2] and no attempt is made in this analysis to remeasure these signs.

Not all of the kinematical quantities needed to evaluate Eqn. 1 for each detected event are well determined. For example, the azimuthal angle of the $\tau$ momentum around the twopion momentum can take on a range of values, restricted by kinematical constraints. Initial state radiation, radiative corrections to the decays $\tau^{\mp} \rightarrow l^{\mp} \bar{\nu} \nu$ and $\tau^{\mp} \rightarrow \pi^{\mp} \pi^{0} \nu$, external bremsstrahlung, and uncertainties of the measured momenta will also modify the evaluation of Eqn. 4 . 
These indeterminancies are taken into account in a likelihood function by forming a weighted sum over all possible kinematical configurations. The weights are derived by assuming that the radiative effects and the resolution of the detector factorize from the Born level matrix element and do not depend on the fit parameters. Formally, the likelihood function is taken to be

$$
L(\Theta \mid \vec{a}):=P(\vec{a} \mid \Theta)=\frac{\int|\mathcal{M}(\vec{\alpha}, \vec{\beta} \mid \Theta)|^{2} \eta(\vec{\alpha}) w(\vec{a}, \vec{\beta} \mid \vec{\alpha}) d \vec{\beta} d \vec{\alpha}}{\int|\mathcal{M}(\vec{\alpha}, \vec{\beta} \mid \Theta)|^{2} \eta(\vec{\alpha}) w(\vec{a}, \vec{\beta} \mid \vec{\alpha}) d \vec{\beta} d \vec{\alpha} d \vec{a}}
$$

where $|\mathcal{M}|^{2}$ is given by Eqn. 4. The vector $\Theta$ represents the set of parameters $\left(\rho, \eta, h_{\nu_{\tau}} \xi, h_{\nu_{\tau}} \xi \delta\right)$ that are determined in the fit. As discussed below, we also include the $\Theta$ dependence of all significant sources of background in the event likelihood. The vector $\vec{a}$ contains all measured quantities, i.e. the momenta of the charged lepton and the two pions. The vector $\vec{\beta}$ contains all unmeasured quantities, such as those associated with the neutrinos, the photons of the initial state bremsstrahlung which mostly escape undetected down the beam pipe, radiated photons in the decay, and photons from external bremsstrahlung. The vector $\vec{\alpha}$ represents the value of the measured quantities before resolution and radiative effects. The weight $w(\vec{a}, \vec{\beta} \mid \vec{\alpha})$ contains all of the resolution and radiative effects, and the integrals over $\vec{\alpha}$ perform convolutions with the detector resolution. The acceptance function of the detector is denoted by $\eta$ and depends only on $\vec{\alpha}$. The denominator in Eqn. 5 ensures that the likelihood integrated over $\vec{a}$ is normalized to unity for all values of $\Theta$.

The integration over the unmeasured quantities is done analytically as far as possible. The remaining integration is performed numerically following a hit or miss approach for the relevant kinematical variables. Hence, a certain number of trials is done for each observed event to generate the unmeasured quantities $\vec{\beta}$ (radiated photons), and the "before radiation and detector resolution" values $\vec{\alpha}$ of the measured quantities, under the hypothesis that the event is a $\tau$ pair. The fraction of trials which are successful, $f_{\text {hit }}$, is a measure of the goodness of the hypothesis. The Monte Carlo integration over the measured quantities in the denominator of Eqn. 5 is done with a full detector simulation. This technique was used in ref. [2]. Here we have applied only minor changes, such as, for example, taking the radiative corrections in the semihadronic decay into account. A full description can be found in ref. 18 .

The effectiveness of this technique has been demonstrated by generating events with the KORALB/TAUOLA [19,20] Monte Carlo program and applying the fit method to these events. The results are compatible with the input within the statistical errors of the test, which are of order $0.01 \%$. These tests have been performed for Standard Model input values as well as for non-Standard Model values. Thus, at the level of accuracy needed here, we have demonstrated that the method is unbiased, and the factorization assumption mentioned above is justified.

\section{DATA SELECTION}

The measurements presented here were performed with the CLEO II detector [21] at the $e^{+} e^{-}$storage ring CESR. The data sample used here was collected in the years between 1990 
and 1994 at center of mass energies around $\sqrt{s}=10 \mathrm{GeV}$. The integrated luminosity is $\approx 3.5 \mathrm{fb}^{-1}$, with about $3.02 \times 10^{6} \tau$ pairs produced.

Events with exactly two charged tracks with a charge sum of zero are selected. Each track must have a momentum greater than $500 \mathrm{MeV} / \mathrm{c}$ and its distance of closest approach to the interaction point in the plane transverse to the beam must be less than $10 \mathrm{~mm}$. The polar angle of each track relative to the beam must fulfill the condition $|\cos \theta|<0.71$. The two tracks are required to be separated by an opening angle of more than $90^{\circ}$.

To suppress non $\tau$ background we require that not more than one of the two tracks has a momentum greater than $85 \%$ of the beam energy. The total visible energy in the electromagnetic calorimeter has to be greater than $20 \%$ and less than $85 \%$ of $\sqrt{s}$. Additionally, the momenta $\vec{p}_{i}$ of the two tracks have to fulfill the condition $\left|\vec{p}_{1}+\vec{p}_{2}\right| /\left(\left|\vec{p}_{1}\right|+\left|\vec{p}_{2}\right|\right)>0.05$ to suppress cosmic rays.

Photons used to reconstruct $\pi^{0}$ mesons are defined as calorimeter showers that are not matched to a charged track, with an energy greater than $50 \mathrm{MeV}$ and a polar angle of $|\cos \theta|<0.71$. To veto against feed across from other $\tau$ decays, we require zero photon-like isolated showers (more than $30 \mathrm{~cm}$ from the closest track projection into the calorimeter) with an energy of more than $75 \mathrm{MeV}$ for polar angles of $|\cos \theta|<0.71$ and an energy of more than $100 \mathrm{MeV}$ in the case of $|\cos \theta|>0.71$.

In the lepton-versus- $\rho$ sample exactly one $\pi^{0}$ is required with $-4<\left(m_{\gamma \gamma}-m_{\pi^{0}}\right) / \sigma_{m_{\gamma \gamma}}<3$, where the mass resolution $\sigma_{m_{\gamma \gamma}}$ is typically between $5 \mathrm{MeV} / \mathrm{c}^{2}$ and $10 \mathrm{MeV} / \mathrm{c}^{2}$ depending on the $\pi^{0}$ energy. The momentum of the reconstructed $\pi^{0}$ has to be greater than $300 \mathrm{MeV} / \mathrm{c}$.

The track further away in angle from the reconstructed $\pi^{0}$ is required to be either an electron or a muon. Tracks are identified as electrons when their momentum and $d E / d x$ information from the tracking system, as well as the energy measurement in the electromagnetic calorimeter, are consistent with the electron hypothesis. Tracks with momenta greater than $1.5 \mathrm{GeV} / \mathrm{c}$ are identified as muons if they match to hits in the muon counters beyond at least three absorption lengths of material.

The invariant mass of the reconstructed $\pi^{0}$ and the charged pion candidate has to satisfy $m_{\pi^{\mp} \pi^{0}}>0.5 \mathrm{GeV} / \mathrm{c}^{2}$. The missing mass $m_{\text {miss }}$ of the event has to fulfill the condition $m_{\text {miss }}>0.1 \sqrt{s}$. Additionally, we require the Monte Carlo success rate $f_{\text {hit }}$ (see previous section) to be at least $>6.6 \%$. After these three cuts the remaining background from Bhabha events, two-photon interactions, and $q \bar{q}$ events is negligible.

In the $\rho$-versus- $\rho$ sample exactly two $\pi^{0}$ mesons are required with $-4<\left(m_{\gamma \gamma}-\right.$ $\left.m_{\pi^{0}}\right) / \sigma_{m_{\gamma \gamma}}<3$. A momentum cut of greater than $300 \mathrm{MeV} / \mathrm{c}$ is applied on the reconstructed $\pi^{0}$ mesons. The $\pi^{0}$ mesons are associated with the charged tracks by their nearness in angle. The invariant mass $m_{\pi^{\mp} \pi^{0}}$ of the $\rho$ meson candidates have to be greater than $0.5 \mathrm{GeV} / \mathrm{c}^{2}$. These cuts are identical to the $l$-vs- $\rho$ selection and suppress feed across from other $\tau$ events. In addition, the missing transverse momentum of the event, $p_{T}$, and $E_{T O T}$, the total visible energy of the observed particles, have to satisfy $p_{T} /\left(\sqrt{s}-E_{T O T}\right)>0.1$, $p_{T} /(\sqrt{s} / 2)>0.075$, and $E_{T O T} / \sqrt{s}>0.3$. We again require $f_{\text {hit }}>6.6 \%$. After these cuts the contribution from non $\tau$ background is negligible.

This selection results in a sample of 66388 accepted events, comprising 33531 candidates in the topology $\left(e^{\mp} \nu \bar{\nu}\right)\left(\pi^{ \pm} \pi^{0} \bar{\nu}\right), 21680$ candidates in $\left(\mu^{\mp} \nu \bar{\nu}\right)\left(\pi^{ \pm} \pi^{0} \bar{\nu}\right)$, and 11177 candidates in $\left(\pi^{\mp} \pi^{0} \nu\right)\left(\pi^{ \pm} \pi^{0} \bar{\nu}\right)$. These numbers of events are in good agreement with expectations based on world average branching ratios [22]. 
TABLE I. Background contribution from other $\tau$ decays.

\begin{tabular}{|c|c|c|c|c|c|}
\hline \multicolumn{2}{|c|}{ electrons 33531 accepted events } & \multicolumn{2}{|c|}{ muons, 21680 accepted events } & \multicolumn{2}{|c|}{$\rho$ mesons, 11177 accepted events } \\
\hline event topology & $\begin{array}{l}\text { estimated } \\
\text { backgr. \% }\end{array}$ & event topology & $\begin{array}{l}\text { estimated } \\
\text { backgr. \% }\end{array}$ & event topology & $\begin{array}{l}\text { estimated } \\
\text { backgr. \% }\end{array}$ \\
\hline$\overline{\left(e^{ \pm} \nu \bar{\nu}\right)\left(\pi^{\mp} \pi^{0} \pi^{0} \nu\right)}$ & $1.78 \pm 0.20$ & $\overline{\left(\mu^{ \pm} \nu \bar{\nu}\right)\left(\pi^{\mp} \pi^{0} \pi^{0} \nu\right)}$ & $1.73 \pm 0.20$ & $\overline{\left(\pi^{ \pm} \pi^{0} \bar{\nu}\right)\left(\pi^{\mp} \pi^{0} \pi^{0} \nu\right)}$ & $3.95 \pm 0.45$ \\
\hline$\left(e^{ \pm} \nu \bar{\nu}\right)\left(K^{\mp} \pi^{0} \nu\right)$ & $1.94 \pm 0.20$ & $\left(\mu^{ \pm} \nu \bar{\nu}\right)\left(K^{\mp} \pi^{0} \nu\right)$ & $2.00 \pm 0.20$ & $\left(\pi^{ \pm} \pi^{0} \bar{\nu}\right)\left(K^{\mp} \pi^{0} \nu\right)$ & $4.31 \pm 0.50$ \\
\hline$\left(\pi^{ \pm} \bar{\nu}\right)\left(\pi^{\mp} \pi^{0} \nu\right)$ & $0.14 \pm 0.03$ & $\left(\pi^{ \pm} \bar{\nu}\right)\left(\pi^{\mp} \pi^{0} \nu\right)$ & $1.29 \pm 0.18$ & & \\
\hline$\left(e^{ \pm} \nu \bar{\nu}\right)\left(\pi^{\mp} \nu\right)$ & $0.13 \pm 0.02$ & $\left(\mu^{ \pm} \nu \bar{\nu}\right)\left(\pi^{\mp} \nu\right)$ & $0.14 \pm 0.03$ & & \\
\hline remaining sources & $0.96 \pm 0.10$ & remaining sources & $0.90 \pm 0.10$ & remaining sources & $2.04 \pm 0.20$ \\
\hline $\bar{\Sigma}$ & $4.95 \pm 0.30$ & $\bar{\Sigma}$ & $6.06 \pm 0.35$ & $\bar{\Sigma}$ & $10.30 \pm 0.70$ \\
\hline
\end{tabular}

In all three samples, $e$-vs- $\rho, \mu$-vs- $\rho$, and $\rho$-vs- $\rho$, the background from non $\tau$ events is insignificant. The background from $\tau$ events is estimated by using the KORALB/TAUOLA Monte Carlo [19,20]. The results are listed in Table [, where the errors reflect statistical, experimental, and theoretical uncertainties. The total contribution from $\tau$ background is about $5 \%$ for $\left(e^{\mp} \nu \bar{\nu}\right)\left(\pi^{ \pm} \pi^{0} \bar{\nu}\right)$, around $6 \%$ for $\left(\mu^{\mp} \nu \bar{\nu}\right)\left(\pi^{ \pm} \pi^{0} \bar{\nu}\right)$, and approximately $10 \%$ for $\left(\pi^{\mp} \pi^{0} \nu\right)\left(\pi^{ \pm} \pi^{0} \bar{\nu}\right)$ events. The "remaining sources" (Table $\mathbb{Q}$ ) are from a variety of modes.

\section{DATA ANALYSIS}

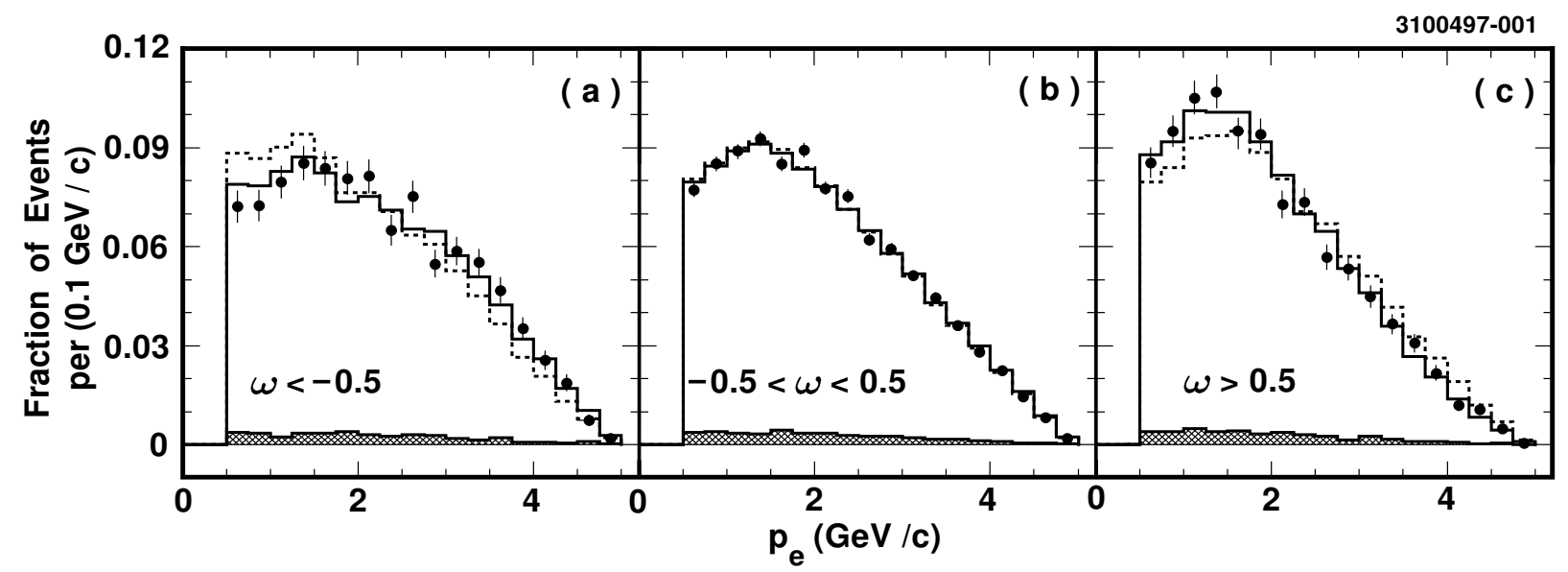

FIG. 1. Electron momentum spectrum in different regions of $\omega$. The variable $\omega$ is sensitive to the spin of the $\tau$ lepton in the decay $\tau^{\mp} \rightarrow \pi^{\mp} \pi^{0} \nu\left(\omega<0 \Rightarrow \tau^{-}\right.$lefthanded, $\omega>0 \Rightarrow \tau^{-}$ righthanded). The data (points with errors) as well as the Monte Carlo expectation for $h_{\nu_{\tau}} \xi=-1$ (solid histograms) show clearly the spin correlation, whereas for $h_{\nu_{\tau}} \xi=0$ (dashed histograms) the two sides of the event are uncorrelated. The hatched histograms show the Monte Carlo predicted background (assuming $h_{\nu_{\tau}} \xi=-1$ ). 


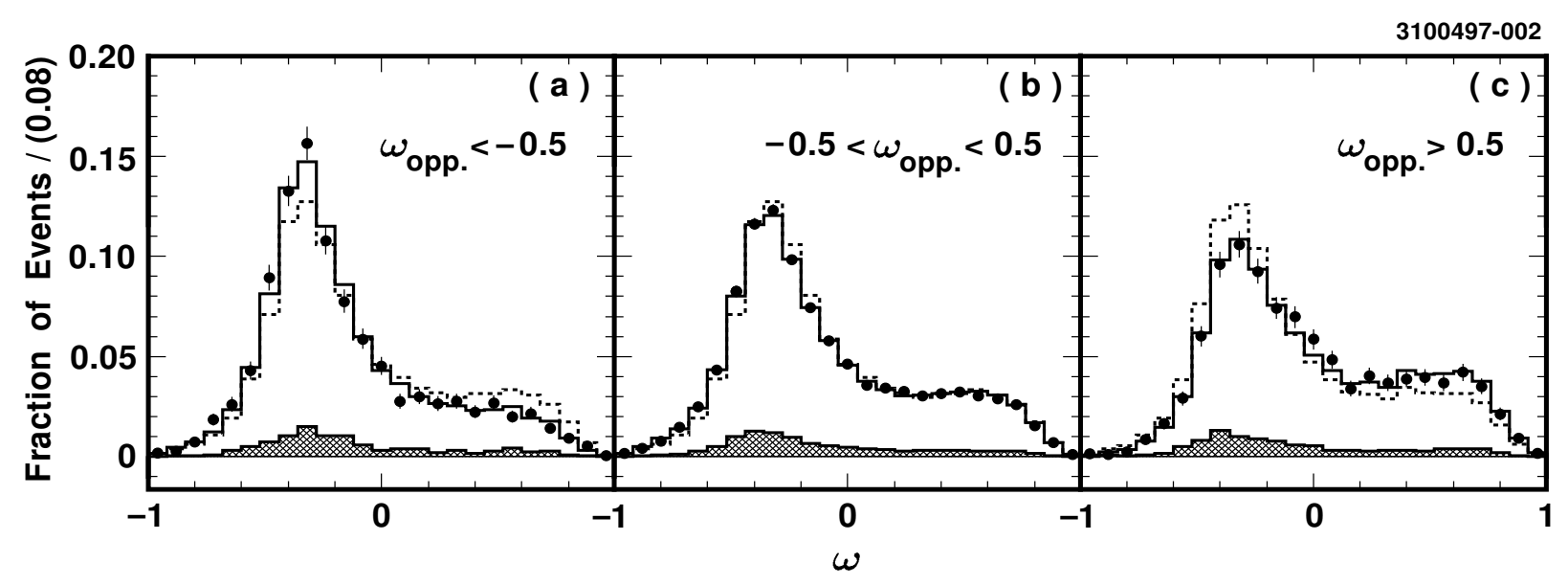

FIG. 2. The $\omega$ spectrum for the $\rho$-vs- $\rho$ sample of one side of the event for different values of $\omega$ from the other side (two entries per event). The data are represented by the dots with error bars. The solid histograms show the Monte Carlo expectation for $h_{\nu_{\tau}}^{2}=1$. The Monte Carlo expectation for $h_{\nu_{\tau}}^{2}=0$ is given by the dashed histograms. Background is indicated by the hatched histograms (assuming $h_{\nu_{\tau}}^{2}=1$ ).

The spin correlation used in this analysis can be most easily illustrated with the spin sensitive variable $\omega$ [23] of the decay $\tau^{\mp} \rightarrow \pi^{\mp} \pi^{0} \nu$. Assuming the Standard Model $V-A$ interaction, the matrix element of this decay in the $\tau$ rest frame is

$$
\left|\mathcal{M}\left(\tau^{\mp} \rightarrow \pi^{\mp} \pi^{0} \nu\right)\right|^{2}=|\overline{\mathcal{M}}|^{2}\left(1 \pm s_{z} H_{z}\right)
$$

where the spin quantization axis $\hat{z}$ is chosen along the flight direction of the $\tau$ in the laboratory frame. The factor $|\overline{\mathcal{M}}|^{2}$ is the spin averaged matrix element. The spin dependent part of the matrix element depends on $H_{z}$ and $s_{z}$, where $H_{z}$ and $s_{z}$ are the $z$-components of the polarimetric vector and the $\tau$ spin vector. For the decay $\tau^{\mp} \rightarrow \pi^{\mp} \pi^{0} \nu, H_{\mu}$ is given by

$$
H_{\mu}=\frac{m_{\tau}\left(2 Q_{\mu}(k Q)-k_{\mu}(Q Q)\right)}{2(k Q)(q Q)-(k q)(Q Q)},
$$

where $k$ denotes the $\tau$ neutrino momentum, $q$ the $\tau$ momentum, and $Q=p_{\pi^{\mp}}-p_{\pi^{0}}$.

As can be seen from Eqn. 6, lefthanded $\tau^{-}$leptons (righthanded $\tau^{+}$leptons) have preferentially negative $H_{z}$ values, whereas righthanded $\tau^{-}$leptons (lefthanded $\tau^{+}$leptons) have preferentially positive $H_{z}$ values. Averaging $H_{z}$ over the kinematically allowed $\tau$ rest frames yields the variable $\omega$.

Fig. 11 shows the dependence of the measured electron momentum spectrum on $\omega$. One clearly sees that for negative $\omega$ values, high momentum leptons are preferred by the data, whereas for positive $\omega$ values, low momentum leptons are preferred. This correlation indicates $h_{\nu_{\tau}} \xi \approx-1$ as expected by the Standard Model. For $h_{\nu_{\tau}} \xi=0$, which is equivalent to zero spin correlation, the lepton momentum spectrum is independent of $\omega$, as can be seen in Fig. 1. The corresponding plots to Fig. 1 for the $\mu$-vs- $\rho$ sample are very similar and are not shown here. Fig. 2 shows for the $\rho$-vs- $\rho$ sample the $\omega$ spectrum of one side of the event for different 
values of $\omega$ from the other side (two entries per event). Again the data favor $h_{\nu_{\tau}}^{2} \approx 1$ in agreement with the Standard Model.

To take the background from other $\tau$ events into account, the fit function of Eqn. 5 is extended to include background

$$
L_{i}=\left(1-\left(\alpha_{1}+\ldots+\alpha_{n}\right)\right) S_{i}+\alpha_{1} B_{1, i}+\ldots+\alpha_{n} B_{n, i}
$$

where $\alpha_{k}$ is the background fraction of the $k$-th background. The function $S_{i}$ is the likelihood of the signal events, given by Eqn. 5. The functions $B_{k, i}$ are the corresponding likelihoods of the backgrounds. For the dominant sources of background, listed in Table $\mathbb{E}$, the functions $B_{k, i}$ include their full dependence on the fit parameters $\Theta$ to avoid bias. The values used in

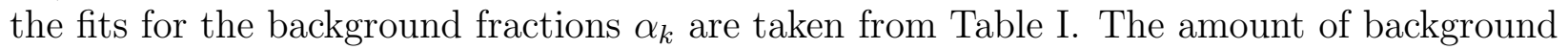
not included in the fit is $\approx 1 \%$ in the $l$-vs- $\rho$ sample and around $2 \%$ in the $\rho$-vs- $\rho$ sample. The effect of this disregarded background ("remaining sources" in Table II) is discussed in the systematic error section.

The hadronic current of the spin analyzer $\tau^{\mp} \rightarrow \pi^{\mp} \pi^{0} \nu$ is very well known. However, the $q^{2}$-dependence of the intermediate resonance structure might be a possible source for uncertainties, especially the contribution of the $\rho^{\prime}$ meson. The $\rho^{\prime}$ contribution is parametrized by $\beta$ [24], and with $e^{+} e^{-}$data [25] $\beta$ is determined [24] to be $\beta=-0.145$. In a recent CLEO measurement [26], where $\tau$ events were used, a value of $\beta=-0.091 \pm 0.009$ is measured. For consistency, we use the latter value together with the mass and width obtained in ref. [26] for the $\rho$ and $\rho^{\prime}$ mesons.

The only fit parameter in the $\rho$-vs- $\rho$ analysis is $h_{\nu_{\tau}}^{2}$. We obtain

$$
h_{\nu_{\tau}}^{2}=0.989 \pm 0.019 \text {. }
$$

In the $e$-vs- $\rho$ analysis we have three fit parameters $\rho, h_{\nu_{\tau}} \xi$, and $h_{\nu_{\tau}} \xi \delta$. We measure the following values:

$$
\rho_{e}=0.747 \pm 0.012 \quad h_{\nu_{\tau}} \xi_{e}=0.973 \pm 0.047 \quad h_{\nu_{\tau}} \xi_{e} \delta_{e}=0.716 \pm 0.031
$$

As mentioned in the introduction, the $l$-vs- $\rho$ sample used here is correlated with the one used in ref. [15]. Because of the special treatment of the low energy muons there, the precision on the Michel parameter $\eta$ reached in ref. [15] is better than the one we might obtain here. Therefore, we do not fit for $\eta$. Instead we fixed $\eta$ to the value determined in ref. [15] of $\eta_{\mu}=0.010 \pm 0.261$ and $\eta_{\mu}=-0.015 \pm 0.091$, where the first result is obtained in the muon sample alone and the second one is the combined result of the muon and electron sample under the assumption $\rho_{e}=\rho_{\mu}$. With the first result we obtain in the $\mu$-vs- $\rho$ analysis

$$
\rho_{\mu}=0.750 \pm 0.017 \quad h_{\nu_{\tau}} \xi_{\mu}=1.048 \pm 0.068 \quad h_{\nu_{\tau}} \xi_{\mu} \delta_{\mu}=0.781 \pm 0.040
$$

Using the second result we measure with the $\mu$-vs- $\rho$ sample

$$
\rho_{\mu}=0.746 \pm 0.017 \quad h_{\nu_{\tau}} \xi_{\mu}=1.043 \pm 0.067 \quad h_{\nu_{\tau}} \xi_{\mu} \delta_{\mu}=0.777 \pm 0.040
$$

All errors shown above are statistical only.

Fixing $\eta$ to the Standard Model value of $\eta=0$ results in a shift of $\rho_{\eta=-0.015}-\rho_{\eta=0}=$ $-0.0029,\left(h_{\nu_{\tau}} \xi\right)_{\eta=-0.015}-\left(h_{\nu_{\tau}} \xi\right)_{\eta=0}=-0.0024$, and $\left(h_{\nu_{\tau}} \xi \delta\right)_{\eta=-0.015}-\left(h_{\nu_{\tau}} \xi \delta\right)_{\eta=0}=-0.0018$. 
TABLE II. Contributions to the systematic error.

\begin{tabular}{|c|c|c|c|c|c|c|c|}
\hline & $\Delta\left(\rho_{e}\right)$ & $\Delta\left(\rho_{\mu}\right)$ & $\Delta\left(h_{\nu_{\tau}} \xi_{e}\right)$ & $\Delta\left(h_{\nu_{\tau}} \xi_{\mu}\right)$ & $\Delta\left(h_{\nu_{\tau}} \xi_{e} \delta_{e}\right)$ & $\Delta\left(h_{\nu_{\tau}} \xi_{\mu} \delta_{\mu}\right)$ & $\Delta\left(h_{\nu_{\tau}}^{2}\right)$ \\
\hline $\begin{array}{l}\text { Monte Carlo } \\
\text { statistics }\end{array}$ & \pm 0.0025 & \pm 0.0028 & \pm 0.0122 & \pm 0.0131 & \pm 0.0090 & \pm 0.0072 & \pm 0.0014 \\
\hline $\begin{array}{l}\text { lepton } \\
\text { identification }\end{array}$ & \pm 0.0006 & \pm 0.0033 & \pm 0.0005 & \pm 0.0016 & \pm 0.0004 & \pm 0.0025 & \\
\hline $\begin{array}{l}\text { acceptance function } \\
\text { of spin analyzer }\end{array}$ & \pm 0.0010 & \pm 0.0015 & \pm 0.0038 & \pm 0.0093 & \pm 0.0036 & \pm 0.0064 & \pm 0.0047 \\
\hline $\begin{array}{l}\text { considered } \\
\text { background }\end{array}$ & \pm 0.0009 & \pm 0.0012 & \pm 0.0018 & \pm 0.0067 & \pm 0.0008 & \pm 0.0023 & \pm 0.0022 \\
\hline $\begin{array}{l}\text { disregarded } \\
\text { background }\end{array}$ & \pm 0.0004 & \pm 0.0009 & \pm 0.0029 & \pm 0.0059 & \pm 0.0011 & \pm 0.0017 & \pm 0.0005 \\
\hline $\begin{array}{l}\text { parameter } \beta \\
\text { of } \rho^{\prime} \text { contribution }\end{array}$ & \pm 0.0002 & \pm 0.0002 & \pm 0.0012 & \pm 0.0025 & \pm 0.0002 & \pm 0.0002 & \pm 0.0010 \\
\hline $\begin{array}{l}\text { Michel parameter } \eta \\
\Delta\left(\eta_{\mu}\right)= \pm 0.091 \text { 15 }\end{array}$ & & \pm 0.0170 & & \pm 0.0144 & & \pm 0.0125 & \\
\hline $\begin{array}{l}\text { Michel parameter } \eta \\
\Delta\left(\eta_{\mu}\right)= \pm 0.261 \text { 15 }\end{array}$ & & \pm 0.0448 & & \pm 0.0417 & & \pm 0.0302 & \\
\hline $\begin{array}{l}\text { detector } \\
\text { resolution }\end{array}$ & \pm 0.0004 & \pm 0.0004 & \pm 0.0006 & \pm 0.0005 & \pm 0.0003 & \pm 0.0004 & \pm 0.0002 \\
\hline radiation & \pm 0.0013 & \pm 0.0011 & \pm 0.0018 & \pm 0.0032 & \pm 0.0021 & \pm 0.0041 & \pm 0.0007 \\
\hline trigger & \pm 0.0017 & \pm 0.0035 & \pm 0.0094 & \pm 0.0123 & \pm 0.0022 & \pm 0.0025 & \pm 0.0011 \\
\hline $\begin{array}{l}\text { total } \\
\Delta\left(\eta_{\mu}\right)= \pm 0.091 \\
\text { total }\end{array}$ & \pm 0.004 & \pm 0.018 & \pm 0.016 & \pm 0.027 & \pm 0.010 & \pm 0.017 & \pm 0.006 \\
\hline$\Delta\left(\eta_{\mu}\right)= \pm 0.261$ & \pm 0.004 & \pm 0.045 & \pm 0.016 & \pm 0.047 & \pm 0.010 & \pm 0.032 & \pm 0.006 \\
\hline
\end{tabular}

The confidence levels of the fits are $73 \%$ in the $e$-vs- $\rho$ analysis, $21 \%$ in the $\mu$-vs- $\rho$ analysis, and $9 \%$ in the $\rho$-vs- $\rho$ analysis. Fig. 3 shows the corresponding likelihood per event distributions. One sees that the data are in good agreement with the best fit model.

Systematic errors arise from statistical errors of the Monte Carlo estimate of the normalization integral, momentum dependence of the lepton identification efficiency, the acceptance function of the $\pi^{\mp} \pi^{0}$ spin analyzer, background, the model for the hadronic current, detector resolution, radiation, and trigger. The different contributions to the systematic error are

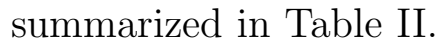

The lepton identification efficiency has been measured, as a function of momentum and polar angle, with independent lepton data samples. The systematic error given in Table [I] arises from the statistical error of this measurement. The acceptance function of the $\pi^{\mp} \pi^{0}$ spin analyzer has been varied via its dependence on the momenta of the two pions and the angle between the two pions. The variations considered have been determined by comparison of Monte Carlo and data. The systematic error due to the considered background has been evaluated by varying the fractions of the different backgrounds in the fit function over a range 
TABLE III. Results. $\rho, \xi$, and $\xi \delta$ denote the combined results. $\rho_{l}, \xi_{l}$, and $\xi_{l} \delta_{l}$ are the results separated for electrons and muons.

\begin{tabular}{|c|c|c|c|c|c|c|}
\hline & world average [22] & this analysis* & \multirow{2}{*}{\multicolumn{4}{|c|}{ correlation coefficients }} \\
\hline$\overline{h_{\nu_{\tau}}}$ & $-1.011 \pm 0.027$ & $-0.995 \pm 0.010 \pm 0.003$ & & & & \\
\hline$\rho$ & $0.742 \pm 0.027$ & $0.747 \pm 0.010 \pm 0.006$ & \multirow{2}{*}{$\begin{array}{l}\kappa(\rho, \xi) \\
\kappa\left(\rho, h_{\nu_{\tau}}\right)\end{array}$} & $=0.046$ & $\kappa(\rho, \xi \delta)$ & $=0.069$ \\
\hline$\xi$ & $1.03 \pm 0.12$ & $1.007 \pm 0.040 \pm 0.015$ & & $=0.000$ & $\kappa(\xi, \xi \delta)$ & $=0.158$ \\
\hline$\xi \delta$ & $0.76 \pm 0.11$ & $0.745 \pm 0.026 \pm 0.009$ & $\kappa\left(\xi, h_{\nu_{\tau}}\right)$ & $=-0.241$ & $\kappa\left(\xi \delta, h_{\nu_{\tau}}\right)$ & $=-0.276$ \\
\hline$\rho_{e}$ & $0.736 \pm 0.028$ & $0.747 \pm 0.012 \pm 0.004$ & $\kappa\left(\rho_{e}, \xi_{e}\right)$ & $=0.046$ & $\kappa\left(\rho_{e}, \xi_{e} \delta_{e}\right)$ & $=0.074$ \\
\hline$\xi_{e}$ & $1.03 \pm 0.25$ & $0.979 \pm 0.048 \pm 0.016$ & $\kappa\left(\rho_{e}, h_{\nu_{\tau}}\right.$ & )$=0.000$ & $\kappa\left(\xi_{e}, \xi_{e} \delta_{e}\right)$ & $=0.216$ \\
\hline$\xi_{e} \delta_{e}$ & $1.11 \pm 0.18$ & $0.720 \pm 0.032 \pm 0.010$ & $\kappa\left(\xi_{e}, h_{\nu_{\tau}}\right)$ & $=-0.194$ & $\kappa\left(\xi_{e} \delta_{e}, h_{\nu_{\tau}}\right)$ & )$=-0.214$ \\
\hline$\rho_{\mu}$ & $0.74 \pm 0.04$ & $0.750 \pm 0.017 \pm 0.045$ & $\kappa\left(\rho_{\mu}, \xi_{\mu}\right)$ & $=0.026$ & $\kappa\left(\rho_{\mu}, \xi_{\mu} \delta_{\mu}\right)$ & $=0.029$ \\
\hline$\xi_{\mu}$ & $1.23 \pm 0.24$ & $1.054 \pm 0.069 \pm 0.047$ & $\kappa\left(\rho_{\mu}, h_{\nu_{\tau}}\right.$ & )$=0.000$ & $\kappa\left(\xi_{\mu}, \xi_{\mu} \delta_{\mu}\right)$ & $=-0.030$ \\
\hline$\xi_{\mu} \delta_{\mu}$ & $0.71 \pm 0.15$ & $0.786 \pm 0.041 \pm 0.032$ & $\kappa\left(\xi_{\mu}, h_{\nu_{\tau}}\right.$ & )$=-0.128$ & $\kappa\left(\xi_{\mu} \delta_{\mu}, h_{\nu_{\tau}}\right.$ & $=-0.152$ \\
\hline & with the sign of $l$ & ormined in & & & & \\
\hline
\end{tabular}

given by statistical, experimental, and theoretical uncertainties. The effect of the disregarded background has been studied using Monte Carlo. The $\rho^{\prime}$ contribution is measured in ref. [26] with an error of $\Delta \beta= \pm 0.009$. Since $\beta$ has model dependencies, and to be conservative, we varied $\beta$ in the range of \pm 0.020 . The systematic error due to $\eta$ has been evaluated by varying $\eta$ in its determined range [15]. The uncertainty in the detector resolution has been estimated by scaling the error matrix of the resolution by a factor of four. The systematic error due to radiation has been obtained by varying the amount of radiation in the fit function by $\pm 10 \%$. The uncertainty in the trigger efficiency arises from the tracking component of the trigger, whereas the uncertainty due to the neutral component of the trigger is negligible. Therefore, the systematic error due to the trigger has been evaluated with a subsample of our data that satisfies the neutral as well as the tracking component of the trigger.

With these systematic errors added in quadrature and using the sign of $h_{\nu_{\tau}}$ determined in ref. [1.2] we obtain the results listed in Table [II]. The results are in agreement with the Standard $V-A$ interaction.

\section{INTERPRETATION AND SUMMARY}

The measurement of $\xi$ and $\delta$ implies that the probability $P_{R}^{\tau}$ (see Eqn. 3) of a righthanded $\tau$ to participate in leptonic $\tau$ decays is $P_{\tau}^{R}<0.044$ at a $90 \%$ confidence level. Separately for electrons and muons, we obtain $P_{\tau}^{R}<0.066$ for the electronic mode and $P_{\tau}^{R}<0.067$ for the muonic mode. Both limits are at a $90 \%$ confidence level.

The $90 \%$ confidence limits on the reduced coupling constants $g_{\epsilon \mu}^{\gamma^{\prime}}=g_{\epsilon \mu}^{\gamma} / \max \left(g_{\epsilon \mu}^{\gamma}\right)$ obtained from the combined results on the Michel parameters (Table III) are plotted in Fig. 4 . Without measuring the helicity of the $\tau$ neutrino in leptonic decays, the $g_{L L}^{S}$ coupling cannot be distinguished from the $g_{L L}^{V}$ coupling. Adding the knowledge of the parameter $\eta$ does not improve the limits. The couplings with a righthanded $\tau, g_{\epsilon R}^{\gamma}$, are mostly constrained by the 
determination of $\xi$ and $\delta$. Additional information comes from the measurement of $\rho$, which allows one to constrain the $g_{R L}^{V}$ and $g_{R L}^{T}$ couplings. Compared to the situation five years ago when only the Michel parameter $\rho$ was measured in $\tau$ decay and no limits on the coupling

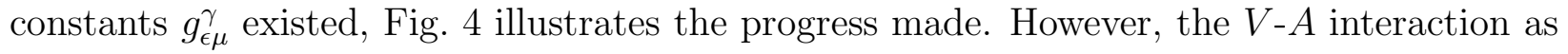
assumed by the Standard Model is still not fully experimentally verified for $\tau$ decays.

More stringent limits can be obtained by restricting the generality of the model. For example, we consider a left-right symmetric model [27] for the electroweak interaction, where the parity violation has its origin in a spontanous symmetry breaking of the left-right symmetry. In addition to the pure lefthanded $W$ boson $W_{L}$ of the Standard Model, such a model assumes a pure righthanded $W$ boson $W_{R}$, where the mass eigenstates $W_{1}$ and $W_{2}$ are in general superpositions of the weak eigenstates $W_{L}$ and $W_{R}$. This model can be parametrized by the mass ratio $\alpha=M_{1} / M_{2}$ of the two bosons $W_{1 / 2}$ and the mixing angle $\zeta$ between $W_{L / R}$. The Standard Model is obtained in the limit $\alpha \rightarrow 0$ and $\zeta \rightarrow 0$.

Fig. 5 shows the one, two, and three $\sigma$ contours for $\alpha$ and $\zeta$ obtained with the combined results on $\rho, \xi, \xi \delta$, and $h_{\nu_{\tau}}$. For $\zeta=0, W_{2}$ is identical with $W_{R}$ and the following limit is obtained on $M_{R}$ :

$$
M_{R}>304 \mathrm{GeV} / \mathrm{c}^{2} \text { at } 90 \% \mathrm{CL}
$$

The mass limit obtained for $\zeta$ free is:

$$
M_{2}>260 \mathrm{GeV} / \mathrm{c}^{2} \text { at } 90 \% \mathrm{CL}
$$

The corresponding likelihood functions are shown in Fig. 6. The limit obtained in muon decay is $M_{2}>406 \mathrm{GeV} / \mathrm{c}^{2}$ [22].

We have also studied the constraints given by our measurement on extensions of the Standard Model with charged Higgs bosons. The $\tau^{-}$lepton and the charged daughter lepton $l^{-}$in leptonic $\tau$ decays mediated by charged Higgs bosons are righthanded [28,29]. Thus, in the general ansatz of Eqn. 1, charged Higgs bosons are represented by the $g_{R R}^{S}$ coupling. From our measurement on $\xi_{\mu}$ and $\xi_{\mu} \delta_{\mu}$ we obtain

$$
M_{H^{ \pm}}>0.91 \times \tan \beta \mathrm{GeV} / \mathrm{c}^{2} \text { at } 90 \% \mathrm{CL},
$$

where $\beta$ is the ratio of vacuum expectation values. The combined limit obtained from this measurement and the recent CLEO measurement of $\eta$ [15] is:

$$
M_{H^{ \pm}}>1.04 \times \tan \beta \mathrm{GeV} / \mathrm{c}^{2} \text { at } 90 \% \mathrm{CL}
$$

All results presented here assume massless $\tau$ neutrinos. We have sudied the kinematical and dynamical effects of a $24 \mathrm{MeV} / \mathrm{c}^{2} \tau$ neutrino on our results and found that for the Michel parameters as well as for the $\tau$ neutrino helicity such a neutrino does not affect the results at the level of our accuracy.

We have presented a precision measurement of the Michel parameters $\rho$, $\xi$, and $\delta$ as well as of the $\tau$ neutrino helicity $h_{\nu_{\tau}}$. The results obtained are consistent with the Standard Model prediction. With the exception of the Michel parameter $\eta$, the CLEO measurement given in ref. [15] is superseded by the results obtained here. Despite the high statistics used, the accuracy of the measurements is still dominated by statistical and not systematic uncertainties, leaving a potential of improving the accuracy of the determination of the Michel parameters in $\tau$ decays at the $B$ factories soon to come into operations, as well as at future $\tau$ factories. 


\section{ACKNOWLEDGEMENTS}

We gratefully acknowledge the effort of the CESR staff in providing us with excellent luminosity and running conditions. J.P.A., J.R.P., and I.P.J.S. thank the NYI program of the NSF, M.S. thanks the PFF program of the NSF, G.E. thanks the Heisenberg Foundation, K.K.G., M.S., H.N.N., T.S., and H.Y. thank the OJI program of DOE, J.R.P., K.H., M.S. and V.S. thank the A.P. Sloan Foundation, A.W. and R.W. thank the Alexander von Humboldt Stiftung, and M.S. thanks Research Corporation for support. This work was supported by the National Science Foundation, the U.S. Department of Energy, and the Natural Sciences and Engineering Research Council of Canada. 


\section{REFERENCES}

[1] ARGUS Collaboration, H. Albrecht et al, Z. Phys. C 5861 (1993).

[2] ARGUS Collaboration, H. Albrecht et al, Phys. Lett. B 349576 (1995).

[3] L. Michel, Proc. Phys. Soc. London A 63514 (1950).

[4] C. Bouchiat and L. Michel, Phys. Rev. 106170 (1957).

[5] L. Okun and A. Rudik, J. Expt. Theor. Phys. (USSR) 32627 (1957) [Sov. Phys. JETP 6 (1957) 520].

[6] F. Scheck, Phys. Rep. 44187 (1978); Leptons, Hadrons, and Nuclei (North-Holland, Amsterdam, 1983).

[7] ARGUS Collaboration, H. Albrecht et al, Phys. Lett. B 246278 (1990).

[8] ARGUS Collaboration, H. Albrecht et al, Phys. Lett. B 316608 (1993).

[9] SLD Collaboration, K. Abe et al, Phys. Rev. Lett. 702515 (1993).

[10] ARGUS Collaboration, H. Albrecht et al, Phys. Lett. B 337383 (1994).

[11] ALEPH Collaboration, D. Buskulic et al, Phys. Lett. B 321168 (1994).

[12] ARGUS Collaboration, H. Albrecht et al, Phys. Lett. B 341441 (1995).

[13] ALEPH Collaboration, D. Buskulic et al, Phys. Lett. B 346379 (1995).

[14] L3 Collaboration, M. Acciari et al, Phys. Lett. B 377313 (1996).

[15] CLEO Collaboration, R. Ammar et al, CLNS-96/1429, submitted to Phys. Rev. Lett.

[16] SLD Collaboration, K. Abe et al, SLAC-PUB-7333, submitted to Phys. Rev. Lett.

[17] S. Jadach and Z. Wa̧s, Acta Phys. Pol. B 151151 (1984).

[18] M. Schmidtler, "Bestimmung der Michelparameter $\xi$ und $\delta$ in leptonischen $\tau$-Zerfällen"

Dr. thesis, Universität Karlsruhe, IEKP-KA/94-16.

[19] S. Jadach and Z. Wạs, Comput. Phys. Commun. 36191 (1985); 64275 (1991); 85453 (1995).

[20] S. Jadach, Z. Wa̧s, R. Decker and J. H. Kühn, Comput. Phys. Commun. 76361 (1993).

[21] CLEO Collaboration, Y. Kubota et al, Nucl. Instr. Meth. A 32066 (1992).

[22] Particle Data Group, R. M. Barnett et al, Phys. Rev. D 541 (1996).

[23] M. Davier, L. Duflot, F. Le Diberder and A. Rougé, Phys. Lett. B 306411 (1993).

[24] J. H. Kühn and A. Santamaria, Z. Phys. C 48445 (1990).

[25] L. M. Barkov et al, Nucl. Phys. B 256365 (1985).

[26] "Fourth Workshop on Tau Lepton Physics", September 1996, Estes Park Colorado, USA. Talk given by J. Urheim representing the CLEO collaboration, "The Hadronic Current in Tau Decay to Two Pseudoscalars".

[27] M. A. B. Bég, R. V. Budny, R. Mohapatra and A. Sirlin, Phys. Rev. Lett. 381252 (1977).

[28] H. E. Haber, G. L. Kane and T. Sterling, Nucl. Phys. B 161493 (1979).

[29] J. F. Gunion and H. E. Haber, Nucl. Phys. B 2721 (1986). 


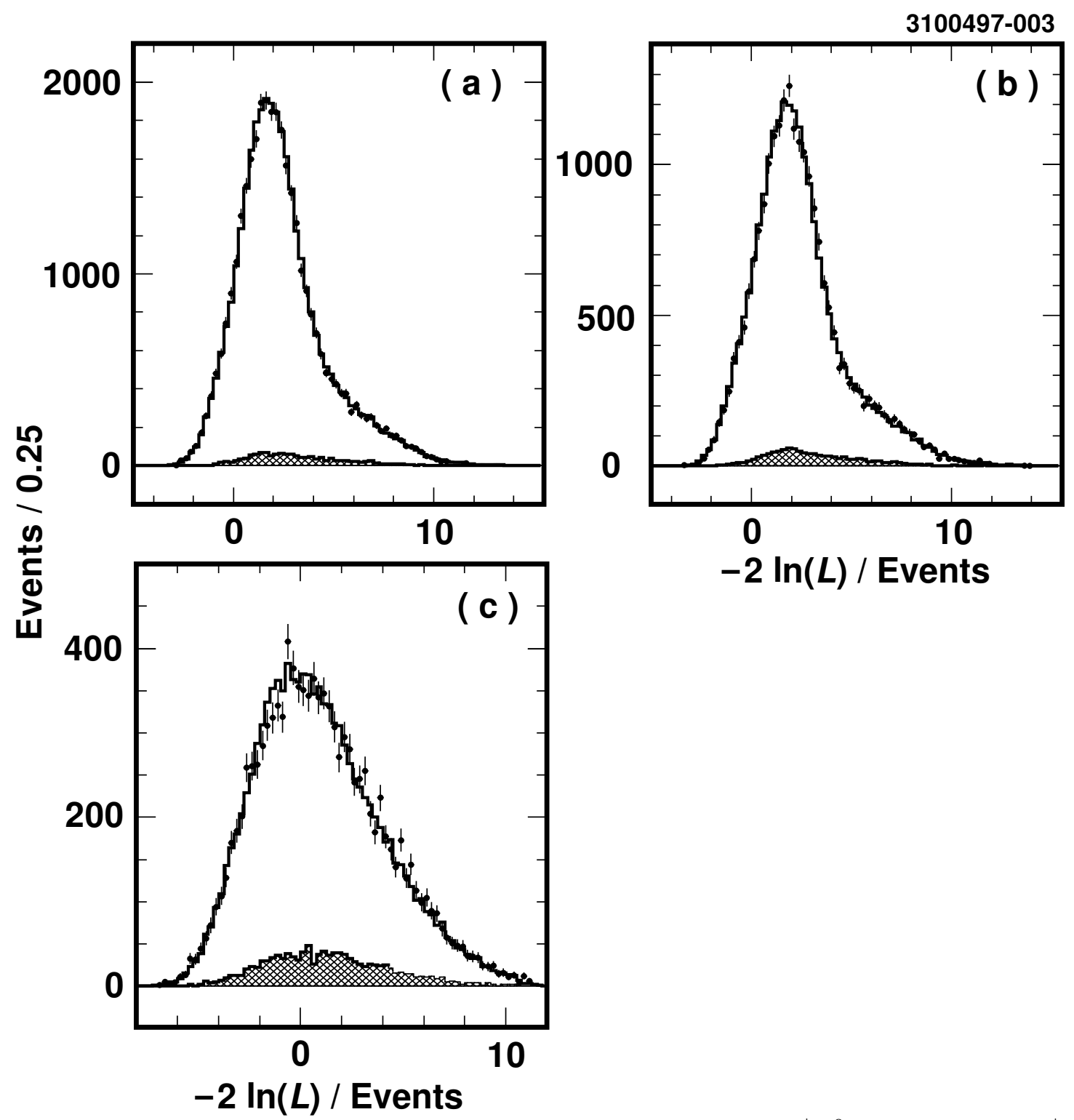

FIG. 3. Contributions of single events to $-2 \ln L$, for (a) $\left(e^{\mp} \nu \bar{\nu}\right)\left(\pi^{ \pm} \pi^{0} \bar{\nu}\right)$, (b) $\left(\mu^{\mp} \nu \bar{\nu}\right)\left(\pi^{ \pm} \pi^{0} \bar{\nu}\right)$, and (c) $\left(\pi^{\mp} \pi^{0} \nu\right)\left(\pi^{ \pm} \pi^{0} \bar{\nu}\right)$ events. Data (dots with error bars) and Monte Carlo (solid histograms) are in good agreement. Background is represented by the hatched histograms. 


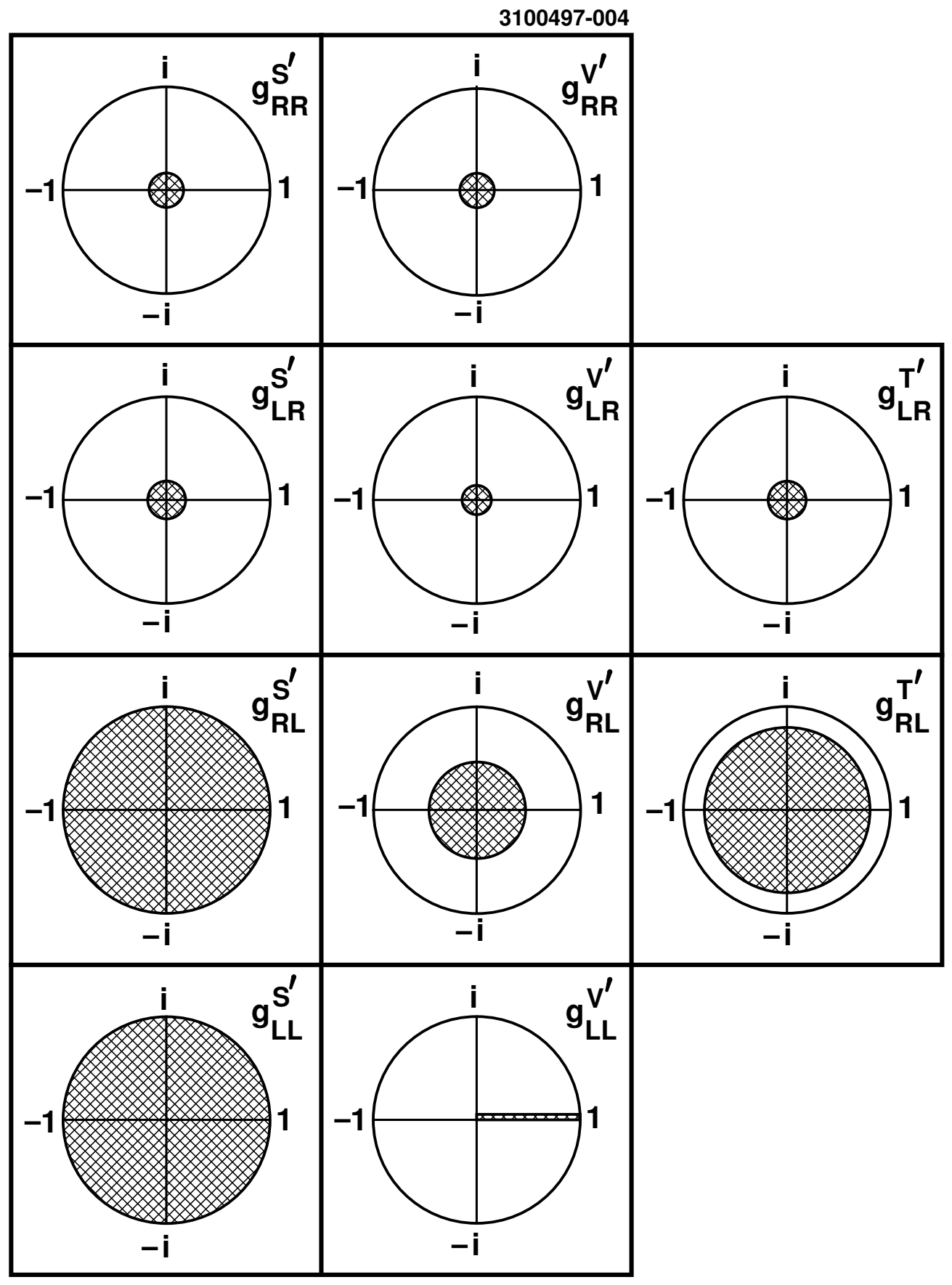

FIG. 4. $90 \%$ confidence limits on the reduced coupling constants $g_{\epsilon \mu}^{\gamma^{\prime}}=g_{\epsilon \mu}^{\gamma} / \max \left(g_{\epsilon \mu}^{\gamma}\right)$. 


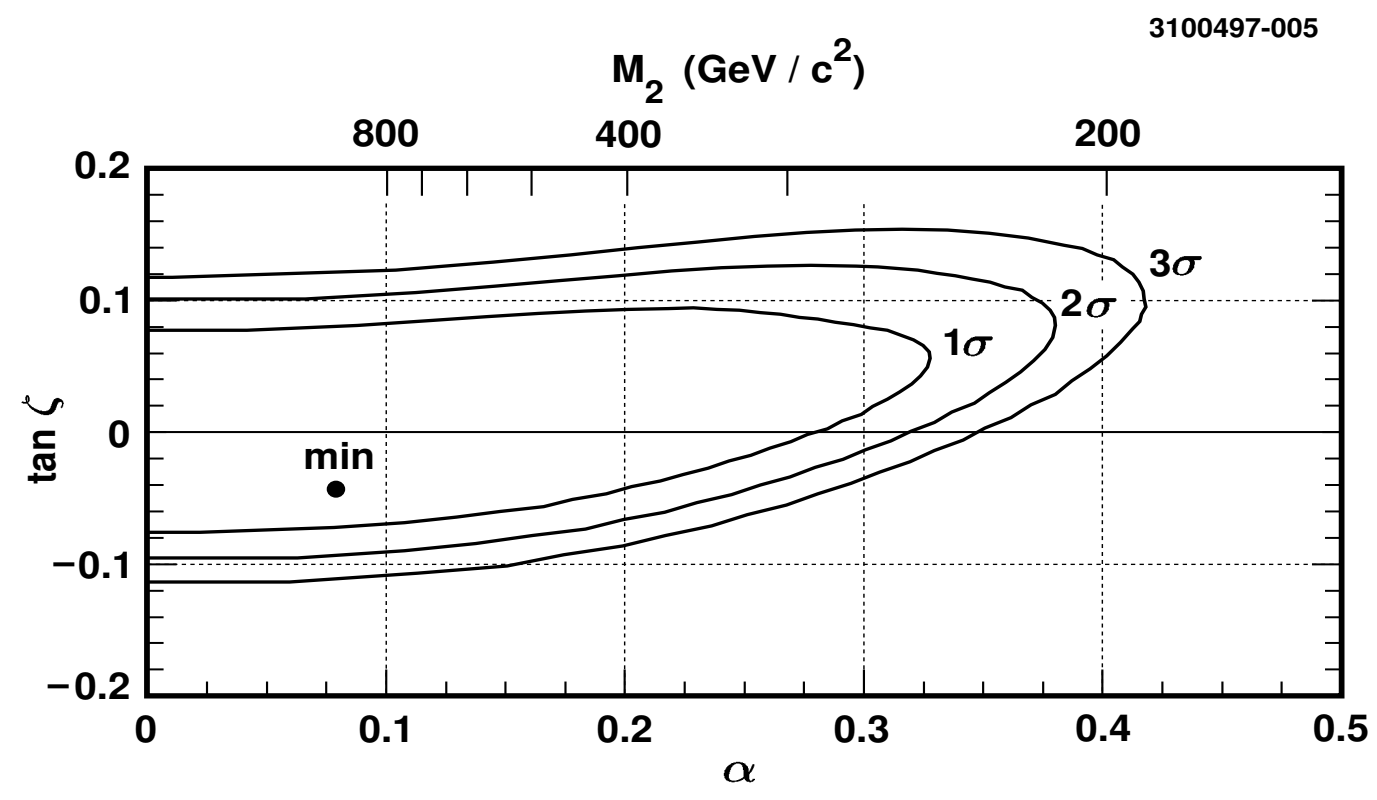

FIG. 5.

Limits on the mass ratio $\alpha$ and the mixing angle $\zeta$ of a left-right symmetric model.

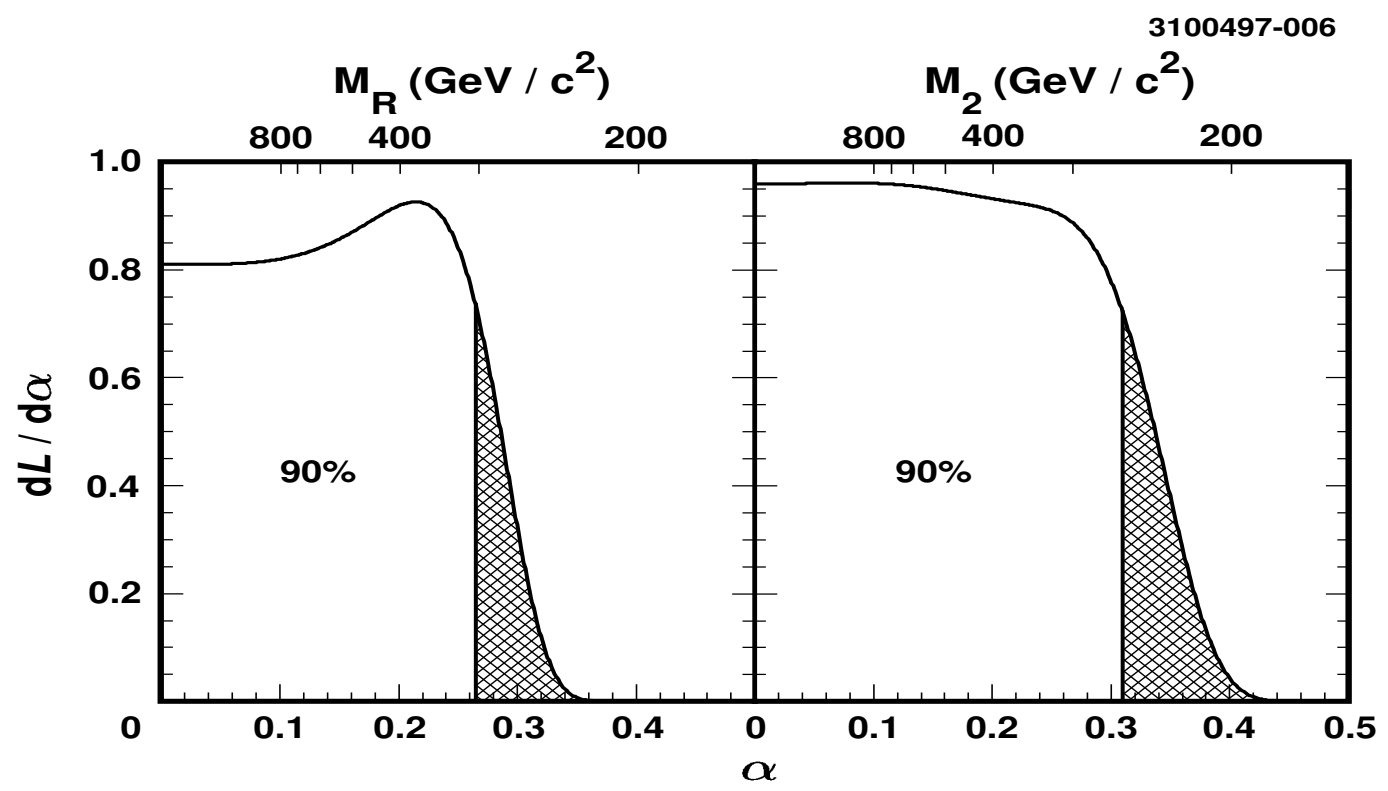

FIG. 6.

$90 \%$ confidence limits on the mass ratio $\alpha$ for (a) $\tan \zeta=0$ and (b) for $\tan \zeta$ free. 